\title{
Fat Feeding in Humans Induces Lipoproteins of Density Less Than 1.006 That Are Enriched in Apolipoprotein [a] and That Cause Lipid Accumulation in Macrophages
}

Thomas P. Bersot, Thomas L. Innerarity, Robert E. Pitas, Stanley C. Rall, Jr., Karl H. Weisgraber, and Robert W. Mahley Gladstone Foundation Laboratories for Cardiovascular Disease, Cardiovascular Research Institute, Departments of Medicine and Pathology, University of California, San Francisco, California 94140-0608

\begin{abstract}
Formula diets containing lard or lard and egg yolks were fed to six normolipidemic volunteers to investigate subsequent changes in the composition of lipoproteins of $d<1.006 \mathrm{~g} / \mathrm{ml}$ and in their ability to bind and be taken up by receptors on mouse macrophages. Both formulas induced the formation of $d<1.006$ lipoproteins that were $\sim 3.5$-fold more active than fasting very low density lipoproteins (VLDL) in binding to the receptor for $\beta$-VLDL on macrophages. Subfractionation of postprandial $\boldsymbol{d}$ $<1.006$ lipoproteins by agarose chromatography yielded two subfractions, fraction I (chylomicron remnants) and fraction II (hepatic VLDL remnants), which bound to receptors on macrophages. However, fraction I lipoproteins induced a 4.6-fold greater increase in macrophage triglyceride content than fraction II lipoproteins or fasting VLDL. Fraction I lipoproteins were enriched in apolipoproteins (apo) B48, E, and [a]. Fraction II lipoproteins lacked apo[a] but possessed apo B100 and apo E. The apo[a] was absent in normal fasting VLDL, but was present in the $d<1.006$ lipoproteins ( $\beta$-VLDL) of fasting individuals with type III hyperlipoproteinemia. The apo[a] from postprandial $d<1.006$ lipoproteins was larger than either of two apo[a] subspecies obtained from lipoprotein (a) $[\mathrm{Lp}(\mathrm{a})]$ isolated at $d=1.05-$ 1.09. However, all three apo[a] subspecies were immunochemically identical and had similar amino acid compositions: all were enriched in proline and contained relatively little lysine, phenylalanine, isoleucine, or leucine. The association of apo[a] with dietary fat-induced fraction I lipoproteins suggests that the previously observed correlation between plasma $L p(a)$ concentrations and premature atherosclerosis may be mediated, in part, by the effect of apo[a] on chylomicron remnant metabolism.
\end{abstract}

\section{Introduction}

The intake of dietary saturated fat and cholesterol in various populations correlates directly with the incidence of coronary heart disease in those populations (1). It has also been shown that experimental animals develop atherosclerosis resulting from hyperlipidemia induced by diets high in fat and cholesterol $(2,3)$. One mechanism thought to be important in diet-induced atherogenesis is the receptor-mediated uptake of lipoproteins by macrophages in the arterial wall, a process that ultimately leads to lipid accumulation and foam cell formation (for review, see References 3 and 4).

Received for publication 16 July 1985.

J. Clin. Invest.

(c) The American Society for Clinical Investigation, Inc.

0021-9738/86/02/0622/09 \$1.00

Volume 77, February 1986, 622-630
The lipoproteins postulated to participate in foam cell formation are referred to as beta-very low density lipoproteins $(\beta$ VLDL) (4-8). The $\beta$-VLDL accumulate in the plasma of animals fed fat and cholesterol and in the plasma of patients with type III hyperlipoproteinemia. The $\beta$-VLDL induced by diet and present in type III patients are of two types: fraction I, of intestinal origin, representing chylomicron remnants, and fraction II, of hepatic origin, representing VLDL remnants (8). When incubated with mouse peritoneal macrophages, both $\beta$-VLDL fractions are capable of causing massive cholesteryl ester accumulation in these cells, giving them the appearance of foam cells (8-12). Other naturally occurring lipoproteins, including low density lipoproteins (LDL), do not induce the same massive cholesteryl ester accumulation in these cells. The uptake of the $\beta$-VLDL is receptor-mediated $(5,6,10,11)$. Van Lenten et al. (13) have shown that postprandial plasma from one human subject contained lipoproteins of $d<1.006 \mathrm{~g} / \mathrm{ml}$ that bound to the $\beta$-VLDL receptor on macrophages. Furthermore, it has been postulated that transiently present postprandial lipoproteins resembling $\beta$-VLDL may occur in humans and may be responsible for the accelerated coronary artery disease associated with dietary fat and cholesterol consumption $(4,7,8,14)$.

Another lipoprotein thought to be atherogenic is lipoprotein (a) $[\mathrm{Lp}(\mathrm{a})]^{1}(15)$, which has been shown to occur in the plasma of fasting subjects, primarily in the density range of 1.05-1.125 $\mathrm{g} / \mathrm{ml}(16,17)$. The $\mathrm{Lp}(\mathrm{a})$ isolated by ultracentrifugation and $\mathrm{pu}-$ rified by column chromatography contains a specific apolipoprotein, apolipoprotein [a] (apo[a]), which has not been detected in any other lipoprotein obtained from the plasma of fasting individuals $(18,19)$. Apo B100 is also a major apolipoprotein constituent of $\mathrm{Lp}(\mathrm{a})$ and probably accounts for the binding of $L p(a)$ to the apo $B, E(L D L)$ receptor $(20,21)$. Levels of $L p(a)$ in plasma vary, ranging from barely detectable to nearly 100 $\mathrm{mg} / \mathrm{dl}$ (22-24). A distinguishing characteristic of $\mathrm{Lp}(\mathrm{a})$ is its high sialic acid content, which is four- to sixfold greater than that of LDL (16). Dietary manipulation, including prolonged fasting, has not been shown to cause changes in the plasma levels of $\mathbf{L p}(a)$ or changes in the distribution of $\mathbf{L p}(\mathrm{a})$ among the lipoprotein classes $(18,23)$. However, there have been no detailed studies of apo[a] in lipoproteins during the acute postprandial state.

The assumed association of $\mathrm{Lp}(\mathrm{a})$ with atherogenesis is based on a direct correlation between plasma $\mathrm{Lp}(\mathrm{a})$ concentrations and the incidence of clinically evident coronary atherosclerosis $(15,23)$. In addition, apo[a] has been identified by immunochemical localization in atherosclerotic lesions of humans (25).

1. Abbreviations used in this paper: apo, apolipoprotein; apo[a], the apolipoprotein that reacts with the specific antisera to the apolipoprotein [a] of either postprandial $d<1.006$ lipoproteins or Lp(a); DME, Dulbecco's modified Eagle's medium; Lp(a), lipoprotein (a) isolated at $d=$ 1.05-1.09. 
The pathophysiology underlying these associations, however, is not understood.

The purpose of the present study was to determine if chylomicrons or chylomicron remnants capable of binding to macrophage receptors in vitro are produced in humans after the ingestion of a single, high-saturated-fat, or high-saturated-fat and high-cholesterol meal, and if lipid accumulation occurs in the macrophages as a consequence of this binding. In these studies, when plasma was procured during the postprandial state, a fraction of the $d<1.006$ lipoproteins similar to chylomicron remnants was isolated and shown to bind avidly to receptors on macrophages, causing triglycerides to accumulate in these cells. The fraction that bound most avidly was enriched in apo[a]. These studies represent the first demonstration of apo[a] in postprandial $d<1.006$ lipoproteins. The previously observed correlation of $\mathrm{Lp}(\mathrm{a})$ with atherosclerosis may be attributable to an association of apo[a] with $d<1.006$ lipoproteins, which are similar, if not identical, to chylomicron remnants.

\section{Methods}

Volunteers. Subjects had appropriate medical histories taken and physical examinations and a laboratory screening were performed to ascertain that they were in good health. Informed consent was obtained.

Dietary studies. The studies were conducted in the metabolic ward of the General Clinical Research Center, San Francisco General Hospital, where the formula diets were prepared. All subjects ate their usual diets prior to the study and abstained from alcohol $3 \mathrm{~d}$ prior to and during the study. Subjects fasted $12 \mathrm{~h}$ before consuming each of the two separate formula meals on consecutive days, day 1 and day 2, of the study. The subjects ate as they wished after the formula meal on day 1 and prior to beginning the 12-h fast required for the next day's study. The formula meal on day 1 consisted of lard, $64 \mathrm{~g}$; dextrose, $32 \mathrm{~g}$; and powdered skim milk, which provided $40 \mathrm{~g}$ of carbohydrate and $27 \mathrm{~g}$ of protein. There were 972 total calories in the formula meal. $12 \mathrm{egg}$ yolks were added to the above formula in preparing the formula meal for day 2 . The egg yolks provided an additional $67 \mathrm{~g}$ of fat, $34 \mathrm{~g}$ of protein, and $\sim 3.0 \mathrm{~g}$ of cholesterol. There were 1,711 calories in the high-fat and high-cholesterol meal.

To obtain sufficient amounts of plasma, $3 \mathrm{U}$ of blood $(\sim 250-300$ $\mathrm{ml} / \mathrm{U}$ ) were taken from each subject using the manual method of plasmapheresis. Each unit of blood required 5-10 min to procure, and the $3 \mathrm{U}$ were collected $\sim 2.5,3.25$, and $4.0 \mathrm{~h}$ after ingestion of the formula meals. The packed cells were reinfused after each unit was drawn, along with a volume of saline equal to that of the plasma that was removed.

Lipoprotein isolation and subfractionation. Aliquots were removed from each unit of plasma to determine plasma lipid and lipoprotein cholesterol concentrations (26). The cholesterol, esterified cholesterol, and triglyceride assays were performed according to standard enzymatic assays (Bio-Dynamics, Boehringer-Mannheim Corp., Indianapolis, IN). The plasma from all $3 \mathrm{U}$ was then pooled, and the $d<1.006$ lipoproteins were isolated by ultracentrifugation in a Beckman $60 \mathrm{Ti}$ rotor (Beckman Instruments, Inc., Palo Alto, CA) at 50,000 rpm for $16 \mathrm{~h}\left(4^{\circ} \mathrm{C}\right)$. A wash was then performed under identical conditions. The $d<1.006$ lipoproteins were then subfractionated by gel permeation chromatography using $4 \%$ agarose as previously described (8). The $d<1.006$ lipoproteins from two individuals (D.R. and E.R.) with type III hyperlipoproteinemia were isolated by ultracentrifugation as described above. Negative staining electron microscopy was used to determine the size of the postprandial $d<1.006$ lipoproteins and the fasting VLDL (27).

The Lp(a) used in these studies was isolated from the plasma of two subjects. One was a normolipidemic individual whose $d>1.006$ fraction demonstrated a pre- $\beta$-migrating band upon electrophoresis. The other was a patient, D.R., with type III hyperlipoproteinemia (8). To isolate the $\mathrm{Lp}(\mathrm{a})$, the plasma density was raised to $1.05 \mathrm{~g} / \mathrm{ml}$, and the plasma was ultracentrifuged for $18 \mathrm{~h}$ at $59,000 \mathrm{rpm}$ in a Beckman $60 \mathrm{Ti}$ rotor. The supernatant fraction was discarded, and the infranatant density was raised to $1.09 \mathrm{~g} / \mathrm{ml}$ and then centrifuged for $24 \mathrm{~h}(59,000 \mathrm{rpm} ; 60 \mathrm{Ti}$ rotor). The $d=1.05-1.09$ fraction that was obtained was then dialyzed against saline-EDTA $(0.01 \%)$ and concentrated to a final protein concentration of $\sim 4 \mathrm{mg} / \mathrm{ml}$ by ultrafiltration under nitrogen pressure. To separate Lp(a) from LDL and high density lipoproteins (HDL), 40-60 $\mathrm{mg}$ of the concentrated $d=1.05-1.09$ fraction was applied to a Pevikon (Mercer Consolidated Corp., Yonkers, NY) preparative electrophoresis block (28). The Lp(a) migrated as a discrete band between the LDL and HDL, neither of which overlapped the $\mathrm{Lp}(\mathrm{a}) .8-10 \mathrm{mg}$ of $\mathrm{Lp}(\mathrm{a})$ protein were obtained from the $40 \mathrm{mg}$ of $d<1.05-1.09$ applied to the block.

Apo E phenotype and plasma concentration measurements. The apo E phenotypes of the subjects were determined by isoelectric focusing of VLDL apolipoproteins (29). Plasma apo E concentrations were determined by radioimmunoassay (30).

Cultured mouse macrophages. Unstimulated peritoneal macrophages were harvested in sterile saline from Swiss-Webster mice as described (9). The macrophages in the peritoneal lavage were identified by size distribution analysis employing a Channelyzer (Coulter Electronics, Inc., Hialeah, FL), and the cells suspended in Dulbecco's modified Eagle's medium (DME) were plated at $5 \times 10^{5}$ macrophages per $16-\mathrm{mm}$ petri dish. After $2 \mathrm{~h}$, the cells were washed three times with DME and then incubated in a humidified $\mathrm{CO}_{2}(7.5 \%)$ incubator overnight in DME containing $20 \%$ fetal calf serum before they were used in an experiment.

Lipoprotein binding and degradation. Radioiodinated $\beta$-VLDL from patients with type III hyperlipoproteinemia $(8,31)$ or from cholesterolfed dogs (32) were prepared with iodine-125 (125I) (33). Binding and cellular proteolytic degradation were determined by previously published procedures $(34,35)$. The details of each experiment are given in the respective figure caption or table legend.

Polyacrylamide gel electrophoresis. Tube gel electrophoresis employing $4 \%$ and $11 \%$ polyacrylamide containing $0.1 \%$ sodium dodecyl sulfate (SDS) and slab gel electrophoresis were performed as previously described $(36,37)$. Electroelution was employed to free apolipoproteins from polyacrylamide gels after preparative slab gel electrophoresis (38).

Immunoblotting. Immunoblotting was performed as described by Daniel et al. (39). After electrophoretic transfer of apolipoproteins from slab gels, the nitrocellulose paper was incubated with monoclonal antibodies specific for apo B100 (18C4) or apo B100 and apo B48 (81A1). The antibody $18 \mathrm{C} 4$, exhibiting apo B100 specificity, has been previously described (40). The antibody $81 \mathrm{~A} 1$ was prepared as described for antibody $18 \mathrm{C} 4$, except apo B48 isolated from human lymph was used as the immunogen instead of human LDL (40). The positive clones were identified by screening against isolated apo B48. All the positive clones produced antibody that cross-reacted against apo $\mathrm{B} 100$, including $81 \mathrm{~A} 1$. A polyclonal antibody specific for apo[a] was also used. This antibody was prepared by immunizing a rabbit with $L p(a)$, harvesting the serum, and then passing this antiserum through an agarose-apo B100 affinity column to remove antibodies to apo B100 (41). After this affinity chromatography was performed, the antiserum failed to react with LDL, apo B100, or VLDL isolated from fasting subjects as determined by Ouchterlony double diffusion and by Western blot analysis.

Cellular accumulation of triglyceride and cholesterol. After the mouse peritoneal macrophages were incubated with various lipoproteins, the cells were harvested, and the amounts of cellular triglyceride, cholesterol, cholesteryl ester, and protein were measured (42). Precise details of the experiment are given in the legend to Table III. Protein was measured according to Lowry et al. (43).

Amino acid composition. Samples to be analyzed were hydrolyzed in $6 \mathrm{~N} \mathrm{HCl}$ for $20 \mathrm{~h}$ at $110^{\circ} \mathrm{C}$ in sealed, evacuated tubes. Amino acid analyses were performed as previously described $(28,44)$.

Sialic acid measurement. The sialic acid content of lipoproteins or apolipoproteins was determined by the thiobarbituric acid assay method (45).

\section{Results}

Description of volunteers. All subjects were normolipidemic (Table I). Three were homozygous for apo E3, two were apo 


\begin{tabular}{|c|c|c|c|c|c|c|c|c|c|c|}
\hline \multirow[b]{3}{*}{ Subject } & \multirow{2}{*}{\multicolumn{2}{|c|}{ Plasma }} & \multicolumn{3}{|c|}{ Lipoprotein } & \multirow{2}{*}{\multicolumn{2}{|c|}{ Apo-E }} & \multirow[b]{3}{*}{ Sex, age } & \multirow[b]{3}{*}{ Weight } & \multirow[b]{3}{*}{ Height } \\
\hline & & & \multicolumn{3}{|c|}{ Cholesterol } & & & & & \\
\hline & Chol & $\mathrm{Tg}$ & VLDL & LDL & HDL & Concn & Phenotype & & & \\
\hline & $m g / d l$ & $m g / d l$ & $m g / d l$ & $m g / d l$ & $m g / d l$ & $m g / d l$ & & & $\mathrm{~kg}$ & $m$ \\
\hline 1 & 163 & 110 & 13 & 104 & 46 & 5.0 & E3/E3 & F, 30 & 69.5 & 1.60 \\
\hline 2 & 135 & 62 & 26 & 68 & 41 & 3.1 & E3/E3 & M, 38 & 75.0 & 1.92 \\
\hline 3 & 134 & 84 & 12 & 84 & 38 & 3.7 & E4/E3 & F, 37 & 67.0 & 1.68 \\
\hline 4 & 144 & 42 & 7 & 94 & 43 & 4.4 & E3/E3 & $M, 36$ & 70.5 & 1.78 \\
\hline 5 & 177 & 153 & 25 & 112 & 40 & 5.2 & E4/E3 & $\mathrm{M}, 44$ & 79.5 & 1.83 \\
\hline 6 & 134 & 76 & 8 & 78 & 48 & 9.4 & $\mathrm{E} 3 / \mathrm{E} 2$ & M, 33 & 81.8 & 1.88 \\
\hline
\end{tabular}

Abbreviations: Chol, cholesterol; $\mathrm{Tg}$, triglycerides; Concn, concentration.

E4/3 heterozygotes, and one was an apo E3/2 heterozygote. The subject with the apo E3/2 phenotype had an elevated plasma apo E concentration. Only one subject (subject 1) exceeded the "normal" weight range (by $0.9 \mathrm{~kg}$ ) indicated in the weight tables of an insurance company. All subjects had detectable Lp(a); however, this apolipoprotein was not measured quantitatively.

Plasma lipid and lipoprotein responses to formula diets. The lipid measurements discussed below were carried out on samples obtained while the subjects were fasting (day 1) and on pooled plasma samples (equal aliquots taken from each of the $3 \mathrm{U}$ of plasma obtained during plasmapheresis) obtained from each individual volunteer in the postprandial state on both day 1 (fat source: lard only) and day 2 (fat source: lard and eggs). Plasma triglyceride concentrations increased proportionately to the amount of dietary triglyceride ingested. Mean values $(\mathrm{mg} / \mathrm{dl})$ \pm SD for the six subjects were: $88 \pm 39$, fasting; $192 \pm 118$, lard diet; and 251 \pm 99 , lard plus eggs diet. Paired $t$ tests indicated that only the fasting values and the values for the lard plus eggs diet were significantly different $(P<0.05)$. Postprandial plasma cholesterol concentrations appeared to decline. However, this may have been due to the large volume of plasma removed by plasmapheresis and replaced with saline. Mean cholesterol values $(\mathrm{mg} / \mathrm{dl})$ were: $148 \pm 18$, fasting; $133 \pm 24$, lard diet; and $116 \pm 18$, lard plus eggs diet. The paired $t$ tests showed that the differences were significant between the values obtained for the fasting and lard plus eggs diet $(P<0.05)$, and the lard diet compared to the lard plus eggs diet $(P<0.05)$. Mean values for LDL cholesterol concentrations (mg/dl) were: $90 \pm 16$, fasting; $82 \pm 19$, lard diet; and $69 \pm 13$, lard plus eggs diet. As with the plasma cholesterol values, paired $t$ tests indicated significant differences between the values obtained for the fasting and lard plus eggs diet $(P$ $<0.05)$, and between the lard diet and the lard plus eggs diet $(P$ $<0.05)$. The HDL cholesterol values $(\mathrm{mg} / \mathrm{dl})$ declined as well: $43 \pm 4$, fasting; $37 \pm 10$, lard diet; and $32 \pm 10$, lard plus eggs diet. Paired $t$ tests showed that the intra-individual decline in HDL cholesterol values was significant only when comparing the fasting and lard plus eggs diet. The plasma concentrations of apo $E$ were not changed by either diet; however, the distribution of apo $\mathrm{E}$ changed considerably in that a greater proportion of apo $\mathrm{E}$ was present in the $d<1.006$ fraction after fat feeding. The proportion of apo $E$ in fasting VLDL was $23 \pm 7.8 \%$ (mean $\pm \mathrm{SD}$ ) of the total plasma apo. $\mathrm{E}$ for the six subjects. After the lard meal, this increased to $30 \pm 10.6 \%$, and after the lard plus eggs meal, the $d<1.006$ fraction contained $45 \pm 12 \%$ of the total plasma apo E. Paired $t$ tests showed a significant difference in the $d<1.006$ content of apo $\mathrm{E}$ in a comparison of the fasting and lard plus eggs values $(P<0.05)$, but not in a comparison of the fasting vs. lard values. When interpreting these results, it is important to keep in mind that the lard plus eggs diet contained not only cholesterol, but also twice as much other fat (from yolk triglycerides and phospholipids) as the lard only diet.

Binding and degradation of fasting VLDL and postprandial $d<1.006$ fractions by mouse peritoneal macrophages. The postprandial $d<1.006$ lipoproteins were much more effective than fasting VLDL in inhibiting both ${ }^{125} \mathrm{I}-\beta$-VLDL binding and uptake (Fig. 1) and degradation (Fig. 1) (a representative experiment). Table II lists the concentrations of fasting VLDL and postprandial $d<1.006$ lipoproteins (either after lard only or lard plus eggs) required to inhibit ${ }^{125} \mathrm{I}-\beta$-VLDL binding to the macrophage receptors by $50 \%$. The ratio of the fasting VLDL to postprandial $d<1.006$ lipoprotein concentrations required to achieve $50 \%$ inhibition of binding provides a means of comparing the data from experiments carried out at different times and with different

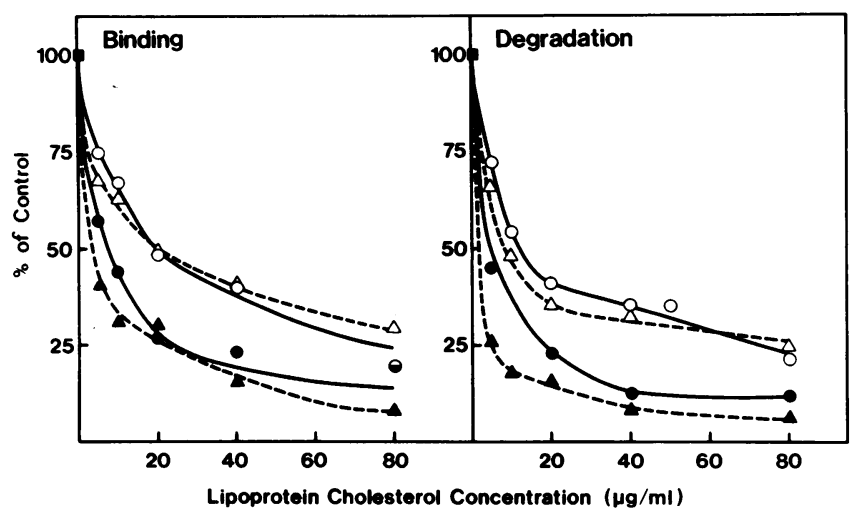

Figure 1. Competitive inhibition of type III ${ }^{125} \mathrm{I}-\beta$-VLDL binding (to mouse macrophages) and degradation by fasting VLDL and postpran$\operatorname{dial} d<1.006$ lipoproteins. After overnight incubation in medium containing $20 \%$ fetal calf serum, each dish received $1 \mu \mathrm{g} / \mathrm{ml}$ (final concentration) of ${ }^{125} \mathrm{I}-\beta$-VLDL and the indicated amount of fasting VLDL $(\Delta, 0)$ or postprandial $d<1.006$ lipoproteins ( $\Delta$, after lard; $\bullet$, after lard and eggs). The added lipoproteins were incubated with the cells for $5 \mathrm{~h}$ at $37^{\circ} \mathrm{C}$, and then the amount of ${ }^{125} \mathrm{I}-\beta$-VLDL bound to the cells and the ${ }^{125} \mathrm{I}-\beta$-VLDL degradative products were determined as described in References 34 and 35 . The cells incubated with ${ }^{125} I-\beta$-VLDL alone (100\% value) bound and internalized $125 \mu \mathrm{g}$ of radioiodinated apolipoprotein/mg of macrophage protein. 
Table II. Concentration of Added $d<1.006$ Lipoproteins Required to Inhibit the Binding of ${ }^{125} I-\beta-V L D L$ to Macrophages by $50 \% *$

\begin{tabular}{|c|c|c|c|c|c|c|c|}
\hline \multirow[b]{2}{*}{ Day of study } & \multicolumn{7}{|c|}{ Subject } \\
\hline & 1 & 2 & 3 & 4 & 5 & $6 \ddagger$ & $6 \ddagger$ \\
\hline & \multicolumn{7}{|c|}{$\mu \mathrm{g}$ of lipoprotein cholesterol/ml } \\
\hline \multicolumn{8}{|l|}{ Day 1} \\
\hline Fasting & 23.0 & 11.5 & 6.8 & 17.0 & 12.5 & 12.5 & 13.5 \\
\hline Lard & 8.0 & 3.5 & 2.5 & 3.6 & 4.4 & 8.9 & 2.4 \\
\hline Ratio of fasting to lard & 2.8 & 3.2 & 3.6 & 4.7 & 2.8 & 1.4 & 5.6 \\
\hline Mean (all ratio values) & 3.4 & & & & & & \\
\hline & \multicolumn{7}{|c|}{$\mu \mathrm{g}$ of lipoprotein cholesterol/ml } \\
\hline \multicolumn{8}{|l|}{ Day 2} \\
\hline Fasting & 25.0 & 14.0 & 8.0 & 7.0 & 11.9 & 26.6 & 12.0 \\
\hline Lard + chol & 5.2 & 1.9 & 2.9 & 5.0 & 3.3 & 11.3 & 7.1 \\
\hline $\begin{array}{l}\text { Ratio of fasting to lard }+ \text { chol } \\
\text { Mean (all ratio values) }\end{array}$ & 4.8 & 7.3 & 1.7 & 1.4 & 3.6 & 2.3 & 1.6 \\
\hline
\end{tabular}

Abbreviation: chol, cholesterol. ${ }^{*}$ The fasting VLDL and postprandial (either after lard or after lard plus cholesterol) $d<1.006$ lipoproteins were obtained from the subjects over a period of $3 \mathrm{wk}$. Two individuals were studied each week. The incubation conditions were as described for Fig. 1 . To compare the results of experiments employing three different pools of macrophages over a 3-wk period of time, the ratio of the fasting VLDL to postprandial $d<1.006$ lipoprotein concentrates required to inhibit ${ }^{125} \mathrm{I}-\beta$-VLDL binding by $50 \%$ was determined for each subject. $\ddagger$ Values for two separate studies.

preparations of both mouse peritoneal macrophages and ${ }^{125} \mathrm{I}-\beta$ VLDL. The mean value of these ratios for the six subjects (comparing fasting and postprandial values) was 3.4 on day 1 , when the fat source was lard, and 3.2 on day 2 , when the fat source was lard and eggs. Thus, the concentration of postprandial $d$ $<1.006$ lipoproteins needed to achieve $50 \%$ inhibition of ${ }^{125} \mathrm{I}$ $\beta$-VLDL binding to macrophages was about one-third of that concentration of fasting VLDL required to achieve the same result.

Subfractionation of postprandial lipoproteins by $4 \%$ agarose gel chromatography. To identify and characterize the postprandial lipoprotein fraction responsible for binding to the macrophage receptor, the postprandial $d<1.006$ lipoproteins from each of the six subjects were separately subfractionated by $4 \%$ agarose gel permeation chromatography. Two incompletely separated fractions, fractions I and II, were obtained (Fig. 2). Fraction I lipoproteins eluted in the void volume and had the following composition (mean values for the six subjects): $83 \%$ triglyceride, $4 \%$ cholesterol, $9 \%$ phospholipid, and $4 \%$ protein. The fraction II lipoproteins contained $56 \%$ triglyceride, $14 \%$ cholesterol, $18 \%$ phospholipid, and $12 \%$ protein. The particle diameters of fraction I lipoproteins ranged from 105 to $280 \mathrm{~nm}$,

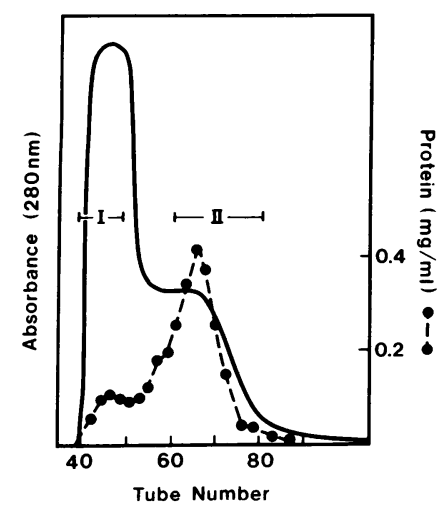

Figure 2. Separation of postprandial (lard meal) $d<1.006$ lipoproteins by $4 \%$ agarose gel chromatography. Postprandial $d<1.006$ lipoprotein protein $(10 \mathrm{mg})$ from subject 6 was applied to a $2.5 \times 100-\mathrm{cm}$ column of Bio-Gel A-15m at $4^{\circ} \mathrm{C}$. Phosphate-buffered saline was pumped through the column at $17 \mathrm{ml} / \mathrm{h}$, and $5-\mathrm{ml}$ aliquots were collected. The protein concentration of the eluate is denoted by the broken line. whereas those of the fraction II particles ranged from 26 to $95 \mathrm{~nm}$.

As shown in a representative experiment, fraction I lipoproteins were more active in binding to the macrophage receptor (Fig. 3). Based on logit-log analysis of the data in Fig. 3, the fraction I lipoproteins were approximately threefold more active than the fraction II lipoproteins in competing with ${ }^{125} \mathrm{I}-\beta$-VLDL for binding. There were no apparent differences between $d$ $<1.006$ lipoproteins (fractions I and II) isolated after lard ingestion and the same fractions obtained after the lard and eggs meal.

Lipid accumulation by macrophages. To determine whether the mass of the cellular lipids increased, the triglyceride, cholesterol, and cholesteryl ester contents of the macrophages were determined after incubation with fasting VLDL or postprandial $d<1.006$ lipoproteins. No measurable triglyceride was detected in cells that had been incubated with culture medium alone,

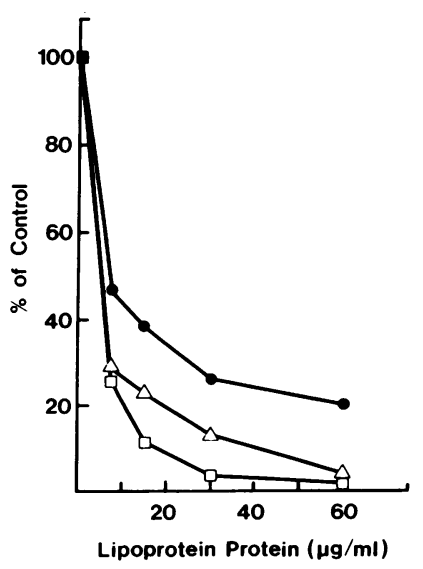

Figure 3. Competitive inhibition of type III ${ }^{125} \mathrm{I}-\beta$-VLDL binding to mouse macrophages by postprandial (after lard) $d$ $<1.006$ subfractions. Macrophages were incubated overnight in medium containing $20 \%$ fetal calf serum, and $2 \mu \mathrm{g} /$ $\mathrm{ml}$ (final concentration) of ${ }^{125} \mathrm{I}$ $\beta$-VLDL were added to each dish. Postprandial $d<1.006$ lipoproteins $(\Delta)$ and fractions I

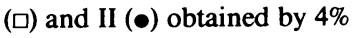
agarose chromatography were then added at the concentrations indicated. After a 5-h incubation, the amount of ${ }^{125} \mathrm{I}-\beta$ VLDL bound and internalized by the cells was determined. Those cells to which no postprandial lipoproteins were added bound $256 \mu \mathrm{g}$ of ${ }^{125} \mathrm{I}-\beta$-VLDL (100\% value). 
LDL, or $\mathrm{Lp}(\mathrm{a})$. However, the postprandial $d<1.006$ lipoproteins increased cellular triglyceride mass to $243.9 \mu \mathrm{g} / \mathrm{mg}$ of cell protein (Table III). Most of this increase was due to fraction I, which induced a 4.6-fold greater increase in cellular triglyceride content than fraction II. There was no detectable increase in cellular cholesteryl ester content as determined either by measuring cholesteryl ester mass (Table III) or by measuring $\left[{ }^{14} \mathrm{C}\right]$ oleate incorporation into cellular cholesteryl esters (data not shown).

Apolipoprotein constituents. No qualitative differences in the apolipoprotein patterns of the six volunteers were apparent when polyacrylamide gel electrophoresis (4\% and $11 \%$ SDS gels) was used to analyze the apolipoproteins of the fasting VLDL and the $d<1.006$ lipoproteins obtained after both test meals (lard and lard plus eggs). Furthermore, no differences in the apolipoprotein patterns (on either $4 \%$ or $11 \%$ gels) of postprandial $d$ $<1.006$ lipoproteins were apparent, regardless of which test meal had been ingested (gels not shown). However, there were notable differences in the patterns of the fasting VLDL and those of the postprandial $d<1.006$ lipoproteins (Fig. 4). On 4\% polyacrylamide gels, the $d<1.006$ lipoproteins isolated from all six volunteers after fat feeding contained a high molecular weight band (Fig. 4, arrow) with an apparent molecular weight greater than that of apo B100. Disulfide reduction of the $d<1.006$ lipoproteins led to the disappearance of this band and the appearance of another band only slightly above apo B100 (gels not shown). Similar results had previously been obtained with apo[a], the apolipoprotein of $\mathrm{Lp}(\mathrm{a})$ that forms a mixed disulfide with apo B100 $(46,47)$. This high molecular weight band was found almost entirely in fraction I of the postprandial $d<1.006$ lipoproteins and was not usually detectable in fraction II (Fig. 4). This band was absent in fasting VLDL, LDL, and HDL; however, a similar high molecular weight band was also observed in the fasting $d$ $<1.006$ lipoproteins ( $\beta$-VLDL) from two individuals with type III hyperlipoproteinemia (apo E2/2; $\mathrm{Arg}_{158} \rightarrow$ Cys variant) (gels not shown).

To confirm that this band represented the apo[a] of $L p(a)$, Western blot analysis employing a polyclonal antibody to $\mathrm{Lp}(\mathrm{a})$ was used. The apo B100 antibodies had been previously immunoadsorbed from this polyclonal antiserum by employing an agarose-apo B100 affinity column. Thus, the antiserum was specific for apo[a]. The apolipoproteins of a postprandial $d<1.006$ preparation, fractions I and II, and LDL were incubated with 2 -mercaptoethanol and then separated by $4 \%$ SDS-polyacryl-

Table III. Lipid Accumulation in Macrophages Incubated with Various Lipoproteins*

\begin{tabular}{lll}
\hline Lipoproteins & Triglyceride & Cholesteryl ester \\
\hline & $\mu g$ of lipid/mg of cell protein \\
Postprandial $d<1.006$ & & \\
$\quad$ lipoproteins & 243.9 & ND \\
Fraction I & 231.4 & ND \\
Fraction II & 49.9 & ND \\
Fasting VLDL & 39.0 & ND \\
LDL & ND & ND \\
Lp(a) & ND & ND \\
No lipoprotein (control) & ND & ND \\
& & \\
\hline
\end{tabular}

Abbreviation: ND, not detectable.

* Each incubation was conducted for $16 \mathrm{~h}$ with the addition of $100 \mu \mathrm{g}$ of lipoprotein triglyceride.

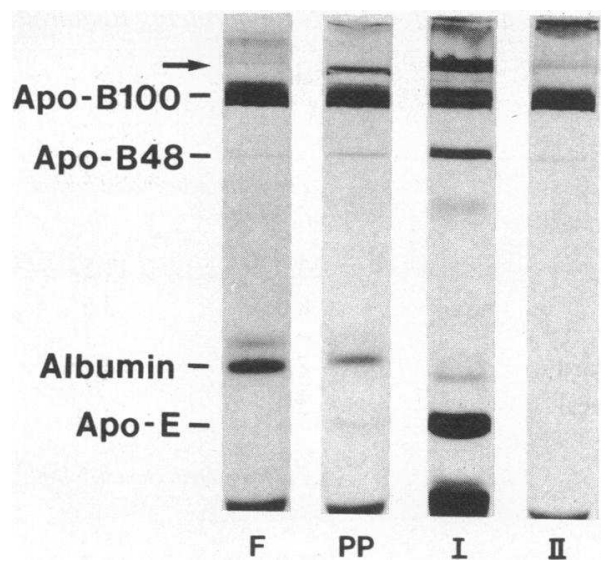

Figure 4. Polyacrylamide gel electrophoresis of fasting VLDL (F), postprandial $d<1.006$ lipoproteins (PP), and fractions I and II (I and II). After delipidation, $30 \mu \mathrm{g}$ of solubilized protein from each lipoprotein was applied to $4 \%$ acrylamide gels. The buffer contained $0.1 \%$ SDS. The arrow indicates a high molecular weight band.

amide slab gel electrophoresis (Fig. 5). The gels were then blotted to nitrocellulose paper, which was subsequently incubated with antibodies to either apo B100 (18C4), apo B100 and apo B48 (81A1), or apo[a] of $\mathrm{Lp}(\mathrm{a})$ (Fig. 5). The $\mathrm{Lp}(\mathrm{a})$-specific apolipoprotein (denoted by the arrow in Fig. 5) reacted only with the anti-Lp(a) serum and was present in the postprandial $d<1.006$

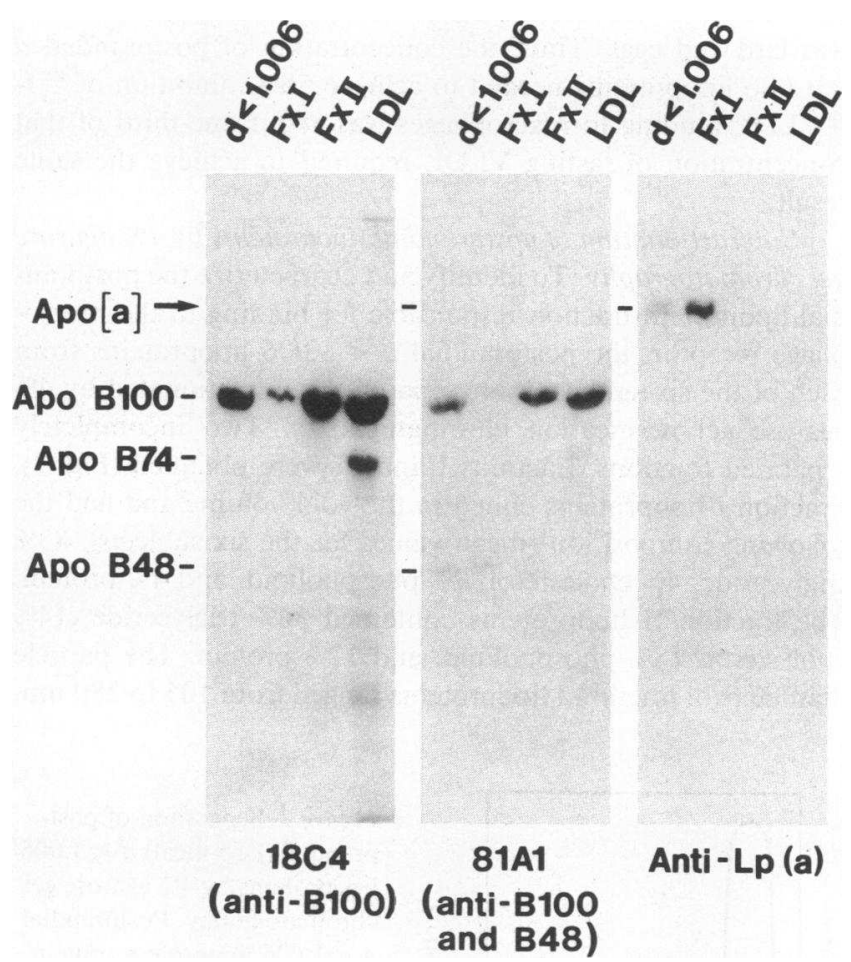

Figure 5. Immunoblot of postprandial $d<1.006$ lipoproteins, fractions I and II (Fx I, Fx II), and LDL. The delipidated apolipoproteins of the indicated lipoproteins were solubilized in buffer containing $0.1 \%$ SDS and 5\% 2-mercaptoethanol. A $10-\mu$ g aliquot of each was applied to the $4 \%$ slab gel. After the gels were electrophoresed, they were blotted with nitrocellulose paper, and the blots were then incubated with the indicated antibodies. A second incubation with ${ }^{125} \mathrm{I}$-antimouse immunoglobulin G, or ${ }^{125} \mathrm{I}$-Protein $\mathrm{A}$ in the case of the anti$L p(a)$, was then followed by exposure of the blots to $x$-ray film. 
and fraction I lipoproteins. Antibodies to apo B100 and apo B48 failed to react with the Lp(a)-specific apolipoprotein (Fig. 5).

The amount of apo[a] present in the postprandial $d<1.006$ lipoproteins varied from one individual to another. However, in all six subjects a readily detectable band corresponding to apo[a] was observed on SDS-polyacrylamide gels of fraction I postprandial $d<1.006$ lipoproteins. Fraction I lipoproteins were also enriched in apo B48 and apo E as compared to fraction II lipoproteins (Fig. 4). There was no apparent difference between the lard or the lard plus eggs meal with regard to the content of apo B48, apo E, or the $\mathrm{Lp}(\mathrm{a})$-specific apolipoproteins in the postprandial $d<1.006$ lipoproteins from the six subjects.

The apo[a] of both postprandial $d<1.006$ lipoproteins and Lp(a) was compared by gradient gel electrophoresis (2-6\% acrylamide). The $\operatorname{Lp}(a)$ used in these studies was from a patient (D.R.) with type III hyperlipoproteinemia. The delipidated apolipoproteins were solubilized in 5\% SDS, and the electrophoresis was carried out with unreduced samples and samples reduced by the addition of 1\% 2-mercaptoethanol (Fig. 6). The postprandial $d<1.006$ lipoproteins contained the high molecular weight apo[a] B100 complex that yielded a lower molecular weight apo[a] upon disulfide reduction, designated apo[a $]_{v}(v$ indicating its association with lipoproteins in the VLDL or $d$ $<1.006$ fraction). Upon reduction, it appeared that the apo B100 of the complex migrated with the apo B100 already present in this lipoprotein. On the other hand, as shown in Fig. 6, the unreduced $\mathrm{Lp}(\mathrm{a})$ isolated from the $d=1.05-1.09$ fraction displayed two bands with mobility intermediate between the postprandial $d<1.006$ apo[a $]_{v} \cdot \mathrm{B} 100$ complex and the apo[a $]_{v}$. Upon reduction, the $\mathrm{Lp}(\mathrm{a})$ revealed two apo[a] bands, designated apo[a $]_{1}$ and apo[a $]_{2}$, as well as apo B100. Both apo[a] $]_{1}$ and apo[a $]_{2}$ of the $\mathrm{Lp}(\mathrm{a})$ were of lower apparent molecular weight than the apo[a] $]_{v}$ of postprandial $d<1.006$ lipoproteins (Fig. 6). In addition, the apo[a] of $d<1.006$ lipoprotein from individuals with type III hyperlipoproteinemia coelectrophoresed with apo[a] $]_{v}$ of postprandial $d<1.006$ lipoproteins (data not shown). The antibodies to apo B100 and Lp(a) were used to demonstrate that the apo[a $]_{1}$, apo[a $]_{2}$, and apo[a $]_{v}$ were recognized by the antibody specific for apo[a], and not by an antibody to apo B100 (Fig. 7).

Isolation of the three apo[a] subspecies was accomplished by preparative $2-6 \%$ gradient gel electrophoresis of the apoli-

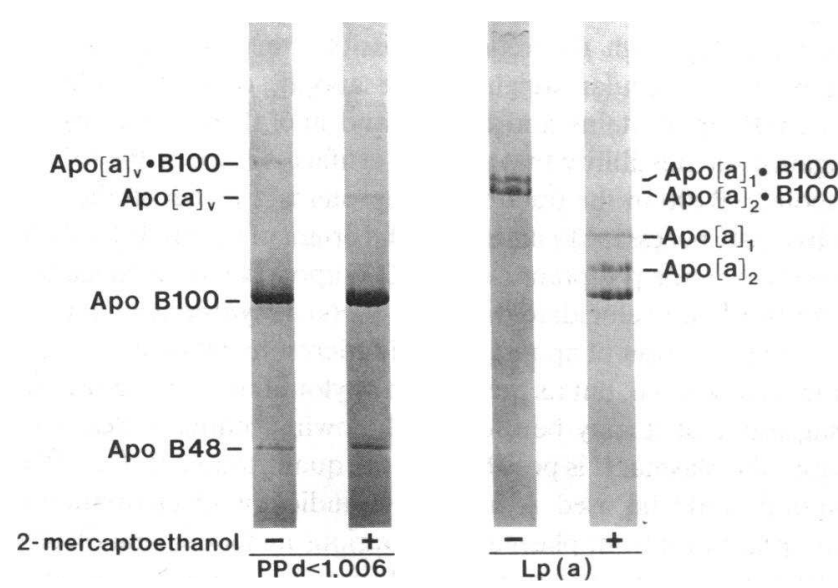

Figure 6. Gradient gel electrophoresis (2-6\% acrylamide) of unreduced $(-)$ and reduced $(+)$ postprandial $d<1.006$ lipoproteins and $L p(a)$ using Coomassie brilliant blue stain.

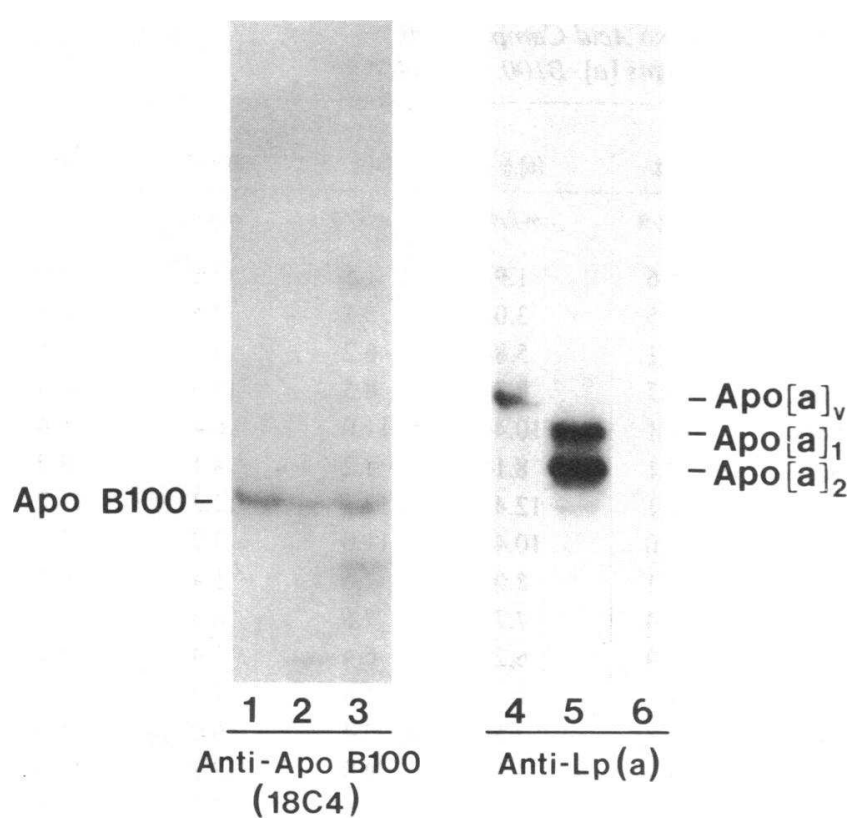

Figure 7. Immunoblots of reduced postprandial $d<1.006$ lipoproteins, Lp(a), and LDL separated by analytical 2-6\% SDS gradient gel electrophoresis. $5 \mu \mathrm{g}$ of the indicated apolipoproteins was applied to the sample wells. For lanes $1-3$, anti-apo B100 (18C4) was used; for lanes 4-6, anti-Lp(a) was employed. Sample identification: lanes 1 and 6. LDL; lanes 2 and 5, Lp(a); lanes 3 and 4, postprandial $d<1.006$ lipoproteins.

poproteins of both $\mathrm{Lp}(\mathrm{a})$ and postprandial $d<1.006$ lipoproteins. The delipidated apolipoproteins were solubilized in 5\% SDS and $1 \%$ 2-mercaptoethanol to reduce the apo[a] - apo B100 disulfide bonds, and the various forms of apo[a] could be clearly separated from the apo B100 on the preparative gels. The three subspecies of apo[a] were electroeluted from the preparative gels, and the amino acid composition and sialic acid content of each subspecies was determined.

The amino acid compositions of the three apo[a] subspecies were nearly identical but differed distinctly from those of apo B100 and apo B48 (Table IV). The apo[a] subspecies were enriched in proline and contained relatively little lysine, as well as small amounts of the hydrophobic residues, isoleucine, leucine, and phenylalanine. The sialic acid content of the three apo[a] subspecies was also determined (Table V). The apo[a] $]_{v}$ from postprandial $d<1.006$ lipoproteins contained the greatest amount of sialic acid, whereas the apo[a $]_{1}$ and apo[a $]_{2}$ of $L p(a)$ had somewhat less sialic acid. These values were four- to fivefold greater than those observed for LDL or for apo B100 and apo B48 obtained from postprandial $d<1.006$ lipoproteins.

\section{Discussion}

These studies provide the first evidence that apo[a] is associated with the postprandial $d<1.006$ lipoproteins induced by fat feeding. In prior studies it appears that postprandial $d<1.006$ lipoproteins were not examined for the presence of apo[a]. The large, triglyceride-rich lipoproteins, representing the chylomicron remnants of the postprandial $d<1.006$ fraction (fraction I), contained virtually all the apo[a]. The smaller postprandial $d$ $<1.006$ lipoproteins, representing VLDL remnants (fraction II), and fasting VLDL did not contain significant quantities of apo[a], as determined by SDS-polyacrylamide gel electrophoresis. 
Table IV. Amino Acid Compositions of Apolipoproteins [a], B100, and B48*

\begin{tabular}{|c|c|c|c|c|c|}
\hline $\begin{array}{l}\text { Amino } \\
\text { acid }\end{array}$ & [a] & {$[a]_{1} \S$} & {$[a]_{2} \S$} & B100\% & B48\% \\
\hline & $\mathrm{mol} / \%$ & $\mathrm{~mol} / \%$ & $\mathrm{~mol} / \%$ & $\mathrm{~mol} / \%$ & $\mathrm{~mol} / \%$ \\
\hline Lys & 1.6 & 1.9 & 1.5 & 7.8 & 7.6 \\
\hline His & 2.5 & 3.0 & 3.1 & 2.5 & 2.5 \\
\hline Arg & 5.1 & 5.8 & 6.2 & 3.2 & 3.5 \\
\hline Asp & 8.7 & 8.7 & 8.5 & 10.9 & 10.9 \\
\hline Thr & 10.1 & 10.4 & 11.0 & 6.4 & 6.4 \\
\hline Ser & 9.1 & 8.1 & 8.2 & 8.1 & 8.2 \\
\hline Glu & 12.1 & 12.4 & 12.3 & 12.2 & 11.7 \\
\hline Pro & 10.0 & 10.4 & 11.0 & 3.7 & 3.9 \\
\hline Gly & 11.1 & 8.0 & 7.6 & 5.4 & 6.7 \\
\hline Ala & 8.4 & 7.7 & 7.6 & 6.3 & 6.6 \\
\hline Val & 5.9 & 6.2 & 6.4 & 5.4 & 5.1 \\
\hline Met & 1.6 & 1.7 & 1.8 & 1.3 & 1.8 \\
\hline Ile & 2.3 & 2.5 & 2.4 & 6.0 & 5.0 \\
\hline Leu & 4.9 & 5.5 & 4.9 & 12.2 & 12.2 \\
\hline Tyr & 5.2 & 6.1 & 6.6 & 3.2 & 3.3 \\
\hline Phe & 1.3 & 1.4 & 1.0 & 5.0 & 4.6 \\
\hline
\end{tabular}

* Values are means for duplicate analyses of apolipoproteins obtained from two separate preparations of postprandial $d<1.006$ lipoproteins and $L p(a)$

$¥$ The apo[a] $]_{\text {v }}$ was obtained from postprandial $d<1.006$ lipoproteins. $\S$ The apo[a $]_{1}$ and apo[a $]_{2}$ were obtained from $\mathrm{Lp}(\mathrm{a})$.

IT The apo B100 and apo B48 were obtained by electroelution from a preparative $2-6 \%$ SDS-polyacrylamide gel to which $10 \mathrm{mg}$ of postprandial $d<1.006$ lipoproteins had been applied.

The fraction I lipoproteins were more active than fraction II lipoproteins in causing triglyceride accumulation within macrophages and also bound more avidly to the receptors on the macrophages. However, it does not appear that these differences relate to the presence of apo[a] in fraction I lipoproteins. Both fractions were bound and internalized, yet only fraction I contained detectable apo[a]. Moreover, the binding of these lipoproteins to macrophage receptors was probably not dependent on the presence of apo B48, because fraction II lipoproteins did bind to macrophage receptors and yet contained no detectable apo B48. It is likely that the difference in receptor binding correlates with the apo $\mathrm{E}$ content of the particles. Fraction I lipo-

Table V. Sialic Acid Contents of Apolipoproteins [a], B100, and B48

\begin{tabular}{ll}
\hline Apolipoprotein or lipoprotein & \multicolumn{1}{c}{ Sialic acid* } \\
\hline & $\mu g / m g$ protein \\
Lp(a) & 27.9 \\
Apo[a $]_{1}$ from $L p(a)$ & 24.4 \\
Apo[a] $]_{2}$ from $L p(a)$ & 34.5 \\
Apo[a] $]_{v}$ from $d<1.006$ lipoproteins & 37.1 \\
Apo B100 from $d<1.006$ lipoproteins & 6.9 \\
Apo B48 from $d<1.006$ lipoproteins & 4.4 \\
LDL & 9.2
\end{tabular}

* Values are means of duplicate samples from two separate preparations of each apolipoprotein or lipoprotein. proteins contained the most apo $\mathrm{E}$ and possessed the greatest binding activity.

The apo[a] $]_{v}$ of postprandial $d<1.006$ lipoproteins is immunochemically identical to the apo[a] $]_{1}$ and apo[a $]_{2}$ of $\operatorname{Lp}(\mathrm{a})$. In addition, the various forms of apo[a] share a similar, unique amino acid composition and are highly sialylated. The apo[a] of postprandial $d<1.006$ lipoproteins is disulfide linked to apo B100, a condition similar to that of apo[a] and apo B100 of $\operatorname{Lp}(a)(46,47)$. However, upon chemical reduction, the apo[a] from the $d<1.006$ lipoproteins has an apparent molecular weight that is greater than that of either the apo[a $]_{1}$ or apo[a $]_{2}$ of $L p(a)$. The apo[a] of $L p(a)$ has previously been shown to exist in two different apparent molecular weight forms (48). It is possible that the apo[a] $]_{v}$ of postprandial $d<1.006$ lipoproteins is a precursor of one or both of these apo[a] subspecies of $L p(a)$, a hypothesis that is currently under study. In addition, the molecular weight differences may reflect differences in the carbohydrate constituents of the various forms of apo[a], independent of sialic acid.

The presence of apo[a] in a complex with apo B100 in the fraction I lipoproteins, which appear to be primarily chylomicron remnants of intestinal origin, requires explanation. Although the data are meager, it appears from previous studies that $L p(a)$ is of hepatic origin and is not synthesized by the intestine (18, 23). However, this needs to be investigated more fully, because prior studies utilized plasma obtained under fasting conditions, and not during alimentary lipemia. The presence of apo B100 in complex with apo[a] suggests several possibilities. First, it is possible that the apo[a $]_{v} \cdot \mathrm{B} 100$ complex is associated with the very large, triglyceride-rich VLDL of hepatic origin that coelute with fraction I lipoproteins of intestinal origin. However, it is difficult to envision that de novo hepatic VLDL synthesis could account for the rapid appearance of the apo[a $]_{v} \cdot \mathrm{B} 100$ complex in the postprandial $d<1.006$ lipoproteins within 2 or $3 \mathrm{~h}$ of the consumption of a high-fat meal. Second, despite the fact that apo B48 is the principal form of apo B synthesized by the intestine, the intestine may synthesize apo B100 that can be complexed with apo[a] in the chylomicrons. A small amount of apo B100 has been detected in human thoracic duct lymph chylomicrons and has been shown to occur in mesenteric lymph chylomicrons synthesized by isolated, perfused segments of rat jejunum $(49,50)$. In addition, it has been shown recently that apo B100 mRNA could be detected in human and rhesus monkey small intestine at significant levels (51). A third possibility is that Lp(a) might fuse with the fraction I lipoproteins, producing an artifact. Although theoretically possible, the difference in the apparent molecular weight of the apo[a] $]_{v}$ of postprandial $d$ $<1.006$ lipoproteins and apo[a] $]_{1}$ and apo[a $]_{2}$ of $\mathrm{Lp}(\mathrm{a})$ argues against the possibility that a fusion artifact is responsible for the apo[a $]_{v} \cdot B 100$ in the fraction I lipoproteins. Further studies are obviously necessary to determine the origin of the apo[a $]_{v} \cdot \mathrm{B} 100$ complex in the postprandial $d<1.006$ lipoproteins. Such studies will more fully elucidate the tissue site(s) of synthesis of apo[a].

The function of apo[a] in chylomicron metabolism also remains unknown, but its presence in chylomicrons and remnants suggests that it may be useful in following remnant clearance from the plasma. It is possible that the quantitation of $d<1.006$ apo[a] could be used as a sensitive indicator of chylomicron disappearance from plasma under various metabolic and dietary perturbations. In addition, apo[a $]_{v}$ may be useful in studying the potential role of transient postprandial lipoproteins in atherogenesis. This unique and highly glycosylated protein may be important in the metabolism of these lipoproteins. 


\section{Acknowledgments}

We thank Jon Hidayat, Kay Arnold, and James McGuire for technical assistance. We appreciate the editorial assistance of Russell Levine, Barbara Allen, and Sally Gullatt Seehafer, the manuscript preparation of Michele Prator, Sylvia Richmond, and Rick Mays, and the graphic arts assistance of James X. Warger and Norma Jean Gargasz.

The work described was carried out in part at the General Clinical Research Center, San Francisco General Hospital Medical Center, with support (RR00083) by the Division of Research Resources, National Institutes of Health.

\section{References}

1. Blackburn, H. 1979. Epidemiological evidence. In Plasma Lipids Optimal Levels for Health. American Health Foundation. Academic Press, Inc., New York. 3-72.

2. Mahley, R. W. 1979. Dietary fat, cholesterol and accelerated atherosclerosis. Atheroscler. Rev. 5:1-34.

3. Mahley, R. W. 1982. Atherogenic hyperlipoproteinemia: the cellular and molecular biology of plasma lipoproteins altered by dietary fat and cholesterol. Med. Clin. N. Am. 66:375-402.

4. Mahley, R. W. 1983. Development of accelerated atherosclerosis: concepts derived from cell biology and animal model studies. Arch. Pathol. Lab. Med. 107:393-399.

5. Mahley, R. W., and T. L. Innerarity. 1983. Lipoprotein receptors and cholesterol homeostasis. Biochim. Biophys. Acta. 737:197-222.

6. Goldstein, J. L., and M. S. Brown. 1977. The low density lipoprotein pathway and its relation to atherosclerosis. Annu. Rev. Biochem. 46: 897-930.

7. Mahley, R. W. 1985. Atherogenic lipoproteins and coronary artery heart disease: concepts derived from recent advances in cellular and molecular biology. Circulation. 72:943-948.

8. Fainaru, M., R. W. Mahley, R. L. Hamilton, and T. L. Innerarity. 1982. Structural and metabolic heterogeneity of $\beta$-very low density lipoproteins from cholesterol-fed dogs and from humans with type III hyperlipoproteinemia. J. Lipid Res. 23:702-714.

9. Goldstein, J. L., Y. K. Ho, M. S. Brown, T. L. Innerarity, and R. W. Mahley. 1980. Cholesteryl ester accumulation in macrophages resulting from receptor-mediated uptake and degradation of hypercholesterolemic canine $\beta$-very low density lipoproteins. J. Biol. Chem. 255: 1839-1848.

10. Mahley, R. W., T. L. Innerarity, M. S. Brown, Y. K. Ho, and J. L. Goldstein. 1980. Cholesteryl ester synthesis in macrophages: stimulation by $\beta$-very low density lipoproteins from cholesterol-fed animals of several species. J. Lipid Res. 21:970-980.

11. Bersot, T. P., T. L. Innerarity, R. W. Mahley, and R. J. Havel. 1983. Cholesteryl ester accumulation in mouse peritoneal macrophages induced by $\beta$-migrating very low density lipoproteins from patients with atypical dysbetalipoproteinemia. J. Clin. Invest. 72:1024-1033.

12. Gianturco, S. H., W. A. Bradley, A. M. Gotto, Jr., J. D. Morrisett, and D. L. Peavy. 1982. Hypertriglyceridemic very low density lipoproteins induce triglyceride synthesis and accumulation in mouse peritoneal macrophages. J. Clin. Invest. 70:168-178.

13. Van Lenten, B. J., A. M. Fogelman, M. M. Hokom, L. Benson, M. E. Haberland, and P. A. Edwards. 1983. Regulation of the uptake and degradation of $\beta$-very low density lipoprotein in human monocyte macrophages. J. Biol. Chem. 258:5151-5157.

14. Zilversmit, D. B. 1973. A proposal linking atherogenesis to the interaction of endothelial lipoprotein lipase with triglyceride-rich lipoproteins. Circ. Res. 33:633-638.

15. Berg, K., G. M. Dahlen, and M. H. Frick. 1974. Lp(a) lipoprotein and pre- $\beta_{1}$-lipoproteins in patients with coronary heart disease. Clin. Genet. 6:230-235.

16. Ehnholm, C., H. Garoff, O. Renkonen, and K. Simons. 1972. Protein and carbohydrate composition of $\mathrm{Lp}(\mathrm{a})$ lipoprotein from human plasma. Biochemistry. 11:3229-3232.

17. Albers, J. J., C.-H. Chin, and F. Aladjem. 1972. Human serum lipoproteins. Evidence for three classes of lipoproteins in $\mathrm{S}_{\mathrm{f}}$ 0-2. Biochemistry. 11:57-63.

18. Krempler, F., G. Kostner, K. Bolzano, and F. Sandhofer. 1979. Lipoprotein (a) is not a metabolic product of other lipoproteins containing apolipoprotein B. Biochim. Biophys. Acta. 575:63-70.

19. Krempler, F., G. M. Kostner, K. Bolzano, and F. Sandhofer. 1980. Turnover of lipoprotein (a) in man. J. Clin. Invest. 65:1483-1490.

20. Utermann, G., and H. Wiegandt. 1969. Darstellung und Charakterisierung eines Lipoproteins mit Antigenwirksamkeit im Lp-System. Humangenetik. 8:39-46.

21. Floren, C.-H., J. J. Albers, and E. L. Bierman. 1981. Uptake of Lp(a) lipoprotein by cultured fibroblasts. Biochem. Biophys. Res. Commun. 102:636-639.

22. Albers, J. J., and W. R. Hazzard. 1974. Immunochemical quantification of human plasma $\mathrm{Lp}(\mathrm{a})$ lipoproteins. Lipids. 9:15-26.

23. Albers, J. J., J. L. Adolphson, and W. R. Hazzard. 1977. Radioimmunoassay of human plasma $\mathrm{Lp}(\mathrm{a})$ lipoprotein. J. Lipid Res. 18: 331-338.

24. Ehnholm, C., H. Garoff, K. Simons, and H. Aro. 1971. Purifcation and quantitation of the human plasma lipoprotein carrying the Lp(a) antigen. Biochim. Biophys. Acta. 236:431-439.

25. Walton, K. W., J. Hitchens, H. Magnani, and M. Khan. 1974. A study of methods of identification and estimation of $\mathrm{Lp}(\mathrm{a})$ lipoprotein and of its significance in health, hyperlipidaemia and atherosclerosis. Atherosclerosis. 20:323-346.

26. Myers, L. H., N. R. Phillips, and R. J. Havel. 1976. Mathematical evaluation of methods for estimation of the concentration of the major lipid components of human serum lipoproteins. J. Lab. Clin. Med. 88: 491-505.

27. Hamilton, R. L., M. C. Williams, C. J. Fielding, and R. J. Havel. 1976. Discoidal bilayer structure of nascent high density lipoproteins from perfused rat liver. J. Clin. Invest. 58:667-680.

28. Mahley, R. W., and K. H. Weisgraber. 1974. An electrophoretic method for the quantitative isolation of human and swine plasma lipoproteins. Biochemistry. 13:1964-1969.

29. Weisgraber, K. H., S. C. Rall, Jr., and R. W. Mahley. 1981. Human $\mathrm{E}$ apoprotein heterogeneity. Cysteine-arginine interchanges in the amino acid sequence of the apo-E isoforms. J. Biol. Chem. 256: 9077-9083.

30. Blum, C. B., L. Aron, and R. Sciacca. 1980. Radioimmunoassay studies of human apolipoprotein E. J. Clin. Invest. 66:1240-1250.

31. Havel, R. J., L. Kotite, J. P. Kane, P. Tun, and T. Bersot. 1983. Atypical familial dysbetalipoproteinemia associated with apolipoprotein phenotype E3/3. J. Clin. Invest. 72:379-387.

32. Mahley, R. W., T. L. Innerarity, K. H. Weisgraber, and D. L. Fry. 1977. Canine hyperlipoproteinemia and atherosclerosis: accumulation of lipid by aortic medial cells in vivo and in vitro. Am. J. Pathol. 87:205-226.

33. Bilheimer, D. W., S. Eisenberg, and R. I. Levy. 1972. The metabolism of very low density lipoproteins. I. Preliminary in vitro and in vivo observations. Biochim. Biophys. Acta. 260:212-221.

34. Goldstein, J. L., Y. K. Ho, S. K. Basu, and M. S. Brown. 1979. Binding site on macrophages that mediates uptake and degradation of acetylated low density lipoprotein, producing massive cholesterol deposition. Proc. Natl. Acad. Sci. USA. 76:333-337.

35. Goldstein, J. L., and M. S. Brown. 1974. Binding and degradation of low density lipoproteins by cultured human fibroblasts. Comparison of cells from a normal subject and from a patient with homozygous familial hypercholesterolemia. J. Biol. Chem. 249:5153-5162.

36. Weber, K., and M. Osborn. 1969. The reliability of molecular weight determinations by dodecyl sulfate-polyacrylamide gel electrophoresis. J. Biol. Chem. 244:4406-4412.

37. Laemmli, U. K. 1970. Cleavage of structural proteins during the assembly of the head of bacteriophage T4. Nature (Lond.). 227:680-685.

38. Stephens, R. E. 1975. High resolution preparative SDS-polyacrylamide gel electrophoresis: fluorescent visualization and electrophoretic elution-concentration of protein bands. Anal. Biochem. 65:369397.

39. Daniel, T. O., W. J. Schneider, J. L. Goldstein, and M. S. Brown. 
1983. Visualization of lipoprotein receptors by ligand blotting. J. Biol. Chem. 258:4606-4611.

40. Hui, D. Y., T. L. Innerarity, and R. W. Mahley. 1984. Defective hepatic lipoprotein receptor binding of $\beta$-very low density lipoproteins from type III hyperlipoproteinemic patients. Importance of apolipoprotein E. J. Biol. Chem. 259:860-869.

41. Egly, J. R., and E. Boschetti. 1983. Practical Guide for Use in Affinity Chromatography and Related Techniques. Société Chimique Pointet-Girard, Villeneuve-La-Garenne, France. 125.

42. Innerarity, T. L., R. E. Pitas, and R. W. Mahley. 1986. Receptorlipoprotein interactions. Methods Enzymol. In press.

43. Lowry, O. H., N. J. Rosebrough, A. L. Farr, and R. J. Randall. 1951. Protein measurement with the Folin phenol reagent. J. Biol. Chem. 193:265-275.

44. Moore, S. 1963. On the determination of cystine as cysteic acid. J. Biol. Chem. 238:235-237.

45. Warren, L. 1959. The thiobarbituric acid assay of sialic acids. $J$. Biol. Chem. 234:1971-1975.

46. Utermann, G., and W. Weber. 1983. Protein composition of
Lp(a) lipoprotein from human plasma. FEBS (Fed. Eur. Biochem. Soc.) Lett. 154:357-361.

47. Gaubatz, J. W., C. Heideman, A. M. Gotto, Jr., J. D. Morrisett, and G. H. Dahlen. 1983. Human plasma lipoprotein [a]. J. Biol. Chem. 258:4582-4589.

48. Fless, G. M., C. A. Rolih, and A. M. Scanu. 1984. Heterogeneity of human plasma lipoprotein(a). Isolation and characterization of the lipoprotein subspecies and their apoproteins. J. Biol. Chem. 259:1147011478

49. Kane, J. P. 1983. Apolipoprotein B: structural and metabolic heterogeneity. Annu. Kev. Physiol. 45:637-650.

50. Wu, A.-L., and H. G. Windmueller. 1981. Variant forms of plasma apolipoprotein B. Hepatic and intestinal biosynthesis and heterogeneous metabolism in the rat. J. Biol. Chem. 256:3615-3618.

51. Knott, T. J., S. C. Rall, Jr., T. L. Innerarity, S. F. Jacobson, M. S. Urdea, B. Levy-Wilson, L. M. Powell, R. J. Pease, R. Eddy, H. Nakai, M. Byers, L. M. Priestley, E. Robertson, L. B. Rall, C. Betsholtz, T. B. Shows, R. W. Mahley, and J. Scott. 1985. Human apolipoprotein B: structure of carboxyl-terminal domains, sites of gene expression, and chromosomal localization. Science (Wash. DC). 230:37-43. 